

\section{Sillares}

Revista de Estudios Históricos

http://sillares.uanl.mx/

Arribo y consolidación del cine sonoro en

Monterrey, 1928-1934

\section{Arrival and consolidation of the sound film in Monterrey, 1928-1934}

Kassandra Donají Sifuentes Zúñiga

Universidad Ciudadana de Nuevo León

orcid.org/0000-0003-4312-6810

Recibido: 18 de agosto de 2021

Aceptado: 10 de octubre de 2021

Publicado: 1 de enero de 2022

Copyright: (C) 2022, Kassandra Donají Sifuentes Zúñiga. This is an open-access article distributed under the terms of Creative Commons Attribution License [CC BY 4.0], which permits unre-stricted use, distribution, and reproduction in any medium, pro-vided the original author and source are credited.

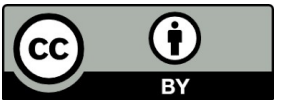

DOI: $\underline{\text { https://doi.org/10.29105/sillares 1.2-4 }}$ 


\title{
Arribo y consolidación del cine sonoro en Monterrey, 1928-1934
}

\author{
Arrival and consolidation of the sound film in Monterrey, \\ 1928-1934
}

Kassandra Donají Sifuentes Zúñiga

Universidad Ciudadana de Nuevo León orcid.org/0000-0003-4312-6810

Resumen: El presente artículo constituye un avance de investigación que tiene como objetivo analizar el impacto sociocultural que tuvo el desarrollo del cine sonoro en la ciudad de Monterrey durante 1928 a 1934, periodo conocido como "El Maximato". A través de un análisis documental, se indagó sobre los aspectos que condicionaron el arribo del cine sonoro y cómo éste llegó a consolidarse entre la sociedad regiomontana determinando la cultura cinematográfica local. Se concluyó que la llamada "Revolución sonora" despertó críticas entre algunos grupos sociales, quienes renegaban de los constantes cambios que el espectáculo presentó. Sin embargo, otros se mostraron entusiasmados logrando la consolidación del cine sonoro, reflejada en la expansión y modificación de salas de cine y en cambios en la estructura de distribución y exhibición cinematográfica. Aunque la aceptación del cine sonoro entre los regiomontanos se presentó de forma gradual, este hecho reforzó el éxito del cine, modificando la forma de asistir y ver películas en la ciudad.

Palabras clave: Cinematografia; Monterrey; cine sonoro; público; exhibición.

Abstract: This article constitutes a work in progress that aims to analyze the socio-cultural impact of the development of the sound Sillares, vol. 1, núm. 2, 2022 
films ("talkies") in the city of Monterrey from 1928 to 1934, a period known as "El Maximato". Through a documentary analysis, I investigated the aspects that conditioned the arrival of the talkies and how they came to be consolidated among the Monterrey society, determining the local film culture. I concluded that the so-called "Sound Revolution" aroused criticism among some social groups, who denied the constant changes that the show presented. However, others were enthusiastic about achieving the consolidation of talkies, reflected in the expansion and modification of movie theaters and changes in the distribution structure and cinematographic exhibition. Although the acceptance of talkies among the people of Monterrey appeared gradually, this fact reinforced the success of the cinema, modifying the way of attending and watching films in the city.

Keywords: Cinematography; Monterrey; sound film; public; exhibition. 


\section{Introducción}

Los primeros estudios académicos en torno al cine se centraron en el análisis de contenidos de películas y de estrellas del celuloide. En este sentido, la historia se ha contado a través de los filmes, tratándolos como testimonios involuntarios o testigos materiales inconscientes de la mentalidad del periodo de su producción, empleando herramientas metodológicas como la hermenéutica, el análisis del discurso y de contenido, la semiótica, entre otras. Pero para muchos teóricos, como Richard Abel, este entretenimiento de masas debe concebirse en términos que van más allá de la producción de textos cinematográficos. ${ }^{1}$

La gran cantidad de estudios relacionados con el análisis de películas ocasionó la necesidad de indagar en nuevos paradigmas, donde los protagónicos fueran la audiencia y sus rituales de consumo, confluyendo aspectos como el contexto histórico, político, económico, las salas de cine, su evolución, la proyección de determinadas películas y el consumo de cada audiencia. ${ }^{2}$ Esto nos permitiría contar una historia cultural y social más amplia y amorfa, más allá del filme, que ayude a comprender las tendencias, factores o condiciones que expliquen la cultura cinematográfica de un lugar.

\footnotetext{
1 Richard Abel, Americaning the Movies and the Movie-Mad Audiences, 1910-1914 (Berkeley y Los Angeles: University of California Press, 2006), 6, citado en Richard Maltby, Explorations in New Cinema History: Approaches and Case Studies (Londres: Blackwell Publishing Ltd, 2011), 9.

2 Philippe Meers, "Metodologías de investigación para la "nueva Historia del cine", en Miscelánea sobre el entorno audiovisual, ed. Francisco Ubierna Gómez y Javier Sierra Sánchez (España: Fragua, 2014), 713.

Sillares, vol. 1, núm. 2, 2022 
A este nuevo paradigma los teóricos lo denominaron "nueva historia del cine". Este enfoque considera los significados culturales y sociales de la asistencia al cine en diferentes épocas basada en experiencias cotidianas, pues según Richard Maltby, la inclusión de experiencias nos muestra detalles más concretos que complementan la microhistoria. ${ }^{3}$ Mediante un análisis histórico, se pretende comprender la cultura cinematográfica de un lugar específico, logrando construir una reflexión acerca de los factores que la determinaron.

A través del siguiente análisis, se cuestiona acerca de qué aspectos de la sociedad regiomontana de la época definieron el desarrollo del cine en la ciudad durante el arribo del sonido al espectáculo. Del problema general se desprenden preguntas específicas que nos ayudarán a entender su influencia en Monterrey: ¿qué implicó para la sociedad regiomontana la llegada del cine sonoro? y ¿cómo ésta determinó el desarrollo subsecuente del cine sonoro en la ciudad?

\section{Antecedentes de investigación}

Como antecedente, este estudio radica en un análisis previo titulado "Implicaciones sociales y culturales relativas al desarrollo del cine en Monterrey, 1898- 1927”, donde examiné el arribo del cinematógrafo a la ciudad y su posterior desarrollo. ${ }^{4}$

3 Maltby, Explorations in New Cinema History: Approaches and Case Studies, 13.

4 Kassandra Sifuentes Zúñiga, "Implicaciones sociales y culturales relativas Sillares, vol. 1, núm. 2, 2022 
Se demostró que, en una primera etapa, periodizada de 1898 a 1910, el espectáculo no tuvo éxito, comparado con otras ciudades del país, ya que se le consideró vulgar y una atracción para las clases más bajas. Durante la segunda etapa estudiada, de 1910 a 1927, el espectáculo cinematográfico se posicionó como uno de los favoritos en la ciudad, desplazando actividades culturales como el teatro y la zarzuela.

De este primer acercamiento se llegó a dos conclusiones. En primer lugar, se encontró que los acontecimientos históricos suscitados en el país determinaron el desarrollo del cine en la ciudad: el arribo del cinematógrafo se presentó en los últimos años del Porfiriato y, en comparación al éxito obtenido en otras ciudades del país, en Monterrey no tuvo el mismo impacto. La dificultad de manejar el aparato trajo consigo una serie de problemas como el requerimiento de energía eléctrica, recintos y técnicos especialistas para su manejo. Esto ocasionó que fuera poco atractivo para los empresarios y el público, que preferiría pasar el tiempo en sus acostumbradas diversiones.

Sin embargo, una coyuntura histórica como la Revolución Mexicana logró que el cine se posicionara como la atracción favorita de la sociedad regiomontana. El desarrollo técnico del aparato había evolucionado y su funcionamiento "avanzado" logró que se capturaran películas sobre los caudillos de la Revolución.

al desarrollo del cine en Monterrey, 1898-1927" (Universidad Autónoma de Nuevo León, 2017).

Sillares, vol. 1, núm. 2, 2022

DOI: https://doi.org/10.29105/sillares1.2-4 
El cine se convirtió en un medio de comunicación con las masas, lo que atrajo a un público curioso por los sucesos bélicos. Fue así que durante este lapso el cine se volvió una actividad económica sustentable para los empresarios, construyendo salas y recintos específicos para este espectáculo.

La segunda conclusión se obtuvo de analizar cómo el cine modificó las prácticas socioculturales de una localidad como Monterrey. Su arribo y su posterior éxito desplazaron actividades de esparcimiento como el teatro o el circo, incluso entre las élites. Por otro lado, los recintos que se construyeron fueron adaptados arquitectónicamente para presentar exhibiciones cinematográficas. Cabe mencionar que un estudio como "Implicaciones sociales y culturales..." no se había realizado anteriormente por los especialistas en cine de la localidad, quienes basaban sus análisis en temporalidades donde la información es vasta, por lo que las contribuciones de este primer estudio ameritaron darle continuidad.

En este trabajo, se estudian específicamente los años de 1928 a 1934, un periodo relevante para el espectáculo en la ciudad debido al arribo del cine sonoro, analizando qué aspectos de la sociedad regiomontana de la época definieron su desarrollo. Del problema general se desprenden preguntas específicas que nos ayudarán a entender la cultura cinematográfica de Monterrey: ¿las coyunturas históricas ocurridas entre 1928 y 1949 condicionaron nuevamente el desarrollo del cine en la ciudad?, ¿existía una Sillares, vol. 1, núm. 2, 2022 
estructura social y económica para la distribución, programación y exhibición de filmes?, y ¿cómo fue la experiencia social de asistir al cine en la ciudad en este periodo?

Apesar de que existen diversos análisis sobre la temporalidad analizada, no se ha estudiado el impacto social y cultural que implicó el desarrollo del cine en la localidad. La intención es reconstruir una historia del cine en Monterrey, desde sus orígenes hasta la actualidad, con una perspectiva histórica-cultural.

\section{El cine sonoro en Monterrey, 1928-1934}

Para Nuevo León, las décadas de 1910 y 1920 constituyeron una época de enorme inestabilidad política. Los gobernadores eran impuestos conforme los planes del presidente en turno, y eso se reflejó en numerosos cambios en el ejecutivo estatal. Durante el periodo revolucionario hubo 19 gobernadores, y en la época posrevolucionaria desfilaron 11 más.

El periodo de transición a la estabilidad inició con Aarón Sáenz, quien llegó a la gubernatura el 4 de octubre de 1927. Abogado, político y militar, simpatizó con las ideas del entonces presidente Álvaro Obregón, quien llegó a nombrarlo Secretario de Relaciones Exteriores en 1923, forjando con él una gran amistad. El apoyo del gobierno federal y de los empresarios locales permitió a Sáenz realizar una importante obra pública, la modernización administrativa y el fortalecimiento del sistema educativo nuevoleonés. 
En 1928, tras el intento de Obregón por conseguir un segundo mandato presidencial, Sáenz obtuvo una licencia para unirse como jefe de campaña del sonorense. En la gubernatura, fue suplido en diversas ocasiones por José Benítez Martínez, también abogado, por Plutarco Elías Calles Chacón (hijo del entonces expresidente Calles), y por Generoso Chapa Garza. Obregón obtuvo el triunfo, pero fue asesinado antes de tomar posesión, y el nombre de Sáenz empezó a sonar fuerte para ocupar el cargo. La jefatura del obregonismo, menciona Segovia y Lajous, recaía casi naturalmente en Sáenz, y su candidatura era casi un hecho consumado. ${ }^{5} \mathrm{El} 3$ denoviembre de 1928, Sáenz nuevamente pidió licencia para retirarse de la gubernatura de Nuevo León por seis meses con el objetivo de concretar una campaña presidencial junto a sus simpatizantes, aunque finalmente no llegaría a ocupar el cargo.

Bajo este contexto político se fue desarrollando la nueva transformación de la industria cinematográfica en la ciudad de Monterrey. Aunque se vio con entusiasmo, el cine sonoro trajo consigo una serie de consecuencias negativas para los empresarios dedicados a este ramo y para los actores. Inicialmente, los magnates de Hollywood se enfrentaron a una barrera idiomática que amenazaba con destruir sus grandes imperios, ya que el fenómeno podía permitir el desarrollo de industrias fílmicas autóctonas en regiones sometidas a su hegemonía. ${ }^{6}$

5 Rafael Segovia y Alejandra Lajous, Historia de la Revolución mexicana, periodo 1928-1934: Los inicios de la Institucionalización, la política del Maximato (México, DF: El Colegio de México, 1978), 30.

6 Rosario Vidal Bonifaz, "Los inicios del cine sonoro y la creación de nuevas Sillares, vol. 1, núm. 2, 2022

DOI: https://doi.org/10.29105/sillares1.2-4 
Algunos productores de Hollywood declararon que la barrera idiomática parecía infranqueable. A pesar de los grandes intentos por combatir el problema, proponiendo distintos métodos como el uso de discos fonográficos, ninguno tuvo valor práctico. Incluso, recurrieron a la grabación de dos filmes, uno interpretado por artistas de habla inglesa y otro con artistas hispanos. Los intentos de llevar al éxito este cine hispano se desvanecieron en poco tiempo, pues el público se llevó una gran decepción al ver que sus artistas favoritos fueron reemplazados por actores hispanos, desmotivando su asistencia al cine. ${ }^{7}$

Los actores y actrices tuvieron que adaptarse a las nuevas formas de realizar películas. Por ejemplo, la actriz estadunidense Marion Davies, tomó clases de canto con el fin de formar parte de esta revolución sonora, pues para todos, sería la primera vez que se escucharía su voz en pantalla. ${ }^{8} \mathrm{Y}$, aunque algunos se rehusaron, como la actriz Dolores del Río, quien expresó a la prensa que no le gustaban las películas parlantes pues el ruido que producían era horrible, pronto no hubo más remedio que adaptarse. ${ }^{9}$

empresas fílmicas en México (1928-1931)”, Revista del Centro de Investigaciones, núm. 29 (2008): 19.

7 Periódico El Porvenir. "El cinematógrafo parlante puede ser considerado como un milagro científicamente patentizado", Monterrey, N.L., 16 de diciembre de $1928,1$.

8 Periódico El Porvenir. "Películas parlantes", Monterrey, N.L., 1 de noviembre de 1928, 2.

9 Periódico El Porvenir. "Dolores llegó ayer a la ciudad de Londres", Monterrey, N.L., 26 de agosto de 1928, 2.

Sillares, vol. 1, núm. 2, 2022

DOI: https://doi.org/10.29105/sillares1.2-4 
Otros advertían que esta nueva forma de hacer cine traería graves consecuencias en sus carreras. Algunos periodistas de la época argumentaban que las películas parlantes amenazaban con causar una revolución radical en la industria cinematográfica. Al dejar el cinematógrafo de ser llamado arte teatral mudo o pantomímico, desaparecerían las estrellas figurantes por la falta de sonido. La nota finaliza señalando que: "Ya no irá el público no más a ver, sino también a oír las películas". ${ }^{10}$

En otra colaboración de El Porvenir, el periodista Alejandro Aragón hizo una fuerte reacción al argumentar que el público solo asistiría a las funciones parlantes con el interés de escuchar a sus actrices y actores favoritos, y menciona:

A usted le gusta el cine hablado por la sencilla razón de que al fin tendrá ocasión de oír hablar a sus artistas favoritos ¿no? ¿Qué interés, preguntamos, podrá encontrarse en esta clase de películas, cuando ni siquiera es el artista popular quien realmente canta o habla, artista por el cual los teatros se ven diariamente concurridos ${ }^{11}$

Las anteriores notas nos demuestran que el acontecimiento en la industria no pasó desapercibido, ya que los periodistas mostraron gran interés hacia el espectáculo cinematográfico. La prensa estuvo atenta a su expansión a nivel internacional, sobre todo por Estados Unidos, donde se relataba cómo era un éxito y la variedad

10 Periódico El Porvenir. "Películas que hacen temblar a Hollywood", Monterrey, N.L., 14 de septiembre de 1928, 3.

11 Periódico El Porvenir. "Últimas revelaciones del cinematógrafo hablado", Monterrey, N.L., 7 de julio de 1929, 4.

Sillares, vol. 1, núm. 2, 2022 
de películas parlantes que se estaban presentando. ${ }^{12}$ Sin embargo, el cine sonoro llegó de forma gradual a diversos puntos de México; contar con estos equipos involucraba modificaciones en los recintos, pues resultó evidente que los teatros donde se exhibían películas parlantes carecían de espacios para acomodar a quienes deseaban verlas y oírlas, sin mencionar la gran inversión en la compra de los aparatos.

En Monterrey, durante 1928, solo se presentaron notas alusivas al suceso, siguiendo con el espectáculo habitual. Algunas funciones siguieron acompañándose de vitrola, orquestas, pianolas e instrumentos que amenizaban la exhibición, como era lo usual. ${ }^{13}$ Para ese mismo año, la ciudad contaba con 10 cines: Teatro Salón Variedades, Teatro Independencia, Teatro Progreso, Teatro Zaragoza, Teatro Obrero, Gran Teatro Rodríguez, Gran Teatro Lírico, Cine Escobedo, Cine Mignon y Salón Terraza B. Reyes. Además estaban aquellos que se localizaban en los barrios y colonias de la ciudad, que no tenían presencia en las carteleras de los periódicos locales, como el Teatro-Cine Libertad, Teatro Cine Madero, Teatro Salón Gloria, entre otros. ${ }^{14}$

La mayoría de las salas de cine pertenecían a la empresa Circuito Rodríguez, liderada por Antonio y Adolfo Rodríguez,

12 Periódico El Porvenir. "Ha tenido éxito el cine parlante en San Antonio", Monterrey, N.L., 4 de febrero de 1928, 2.

13 Periódico El Porvenir. "Vitrola", Monterrey, N.L., 5 de febrero de 1928, 3. 14 Archivo Histórico Municipal de Monterrey (en adelante AHMM), Actas de Cabildo, Fondo Monterrey Contemporáneo, vol. 999, exp. 1928/006, 25 de enero de 1928, f. 5.

Sillares, vol. 1, núm. 2, 2022

175

DOI: https://doi.org/10.29105/sillares1.2-4 
quienes desde 1904 ejercieron una gran labor en torno al espectáculo cinematográfico. Ambos se encargaron de equipar las salas de la ciudad con diversas proyecciones nacionales e internacionales, así como encargarse de salas en el norte del país: Coahuila, Tamaulipas, Zacatecas y San Luis Potosí.

La empresa se encargó de comprar películas por rollo o metro para proyectar a la población regiomontana. En una entrevista, mencionaron que hacia 1910 los filmes europeos eran los favoritos del público regiomontano, pero que en 1914 se dejaron de importar debido a la Primera Guerra Mundial. Ante la situación, se optó por adquirir películas estadounidenses, volviéndose las preferidas entre la audiencia. Los filmes norteamericanos se exhibían en veladas especiales llamadas Premiere, proyectando cintas como Los Perros Contrabandistas (s.f.) y La lámpara de Aladino (s.f.), entre otros. El empresario recordó que después de la guerra se volvió a comprar películas francesas e italianas, pero no volvieron a tener el mismo éxito. ${ }^{15}$ Para esta época, su mercado había crecido y se exhibían películas como La cabaña del Tío Tom, considerada por la prensa como la película más sentimental que se había realizado. ${ }^{16}$

Mientras la inestabilidad política en México se trataba con soluciones a corto plazo, y el cine sonoro se consolidaba, en el mundo se presentaba un problema mayúsculo: la Gran

15 Periódico El Porvenir. Monterrey, N.L., 10 de septiembre de 1943, 7

16 Periódico El Porvenir. "La cabaña del Tío Tom es la película más sentimental que se ha hecho", Monterrey, N.L., 3 de agosto de 1928, 5.

Sillares, vol. 1, núm. 2, 2022

176

DOI: https://doi.org/10.29105/sillares1.2-4 
Depresión. La ola de prosperidad que inundaba el mundo capitalista se interrumpió en octubre de 1929, con el hundimiento de la bolsa de Nueva York. El 29 de ese mes cayeron en picada las cotizaciones bursátiles, poniendo fin a la especulación. La crisis de la bolsa siguió profundizándose en los años sucesivos hasta tocar fondo en 1933. Las consecuencias financieras del hundimiento de la bolsa se unieron a una fuerte reducción del consumo, de forma que la economía norteamericana entró en una recesión generalizada. Una consecuencia fue el rápido aumento del desempleo, hambrunas y el suicidio de algunas personas.

A pesar de la situación económica que se enfrentaba, la crisis no frenó la labor de los empresarios locales de espectáculos, en contraste con otros sucesos históricos que se habían presentado, como la Primera Guerra Mundial o la Revolución Mexicana. En 1929, en el mes de junio, se anunció la película La Ultima canción (1929) de Warner Bros., interpretada por el ya famoso Al Jolson. Para la exhibición, el Circuito Rodríguez logró que se instalaran aparatos en el Teatro Independencia con ayuda de ingenieros y especialistas en el tema, quienes brindaron instrucciones a los operarios para un manejo adecuado. ${ }^{17}$

El estreno de la película entusiasmó a todas las clases sociales, pues según la prensa, era la primera película hablada que se presentaba en la ciudad. ${ }^{18}$ Incluso, la prensa realizaba notas 17 Periódico El Porvenir. "Se acerca el estreno de la cinta hablada La última canción, marca Wagner Brothers por Al Jolson”, Monterrey, N.L., 25 de junio de 1929,8 .

18 Periódico El Porvenir. "Ya se acerca el estreno de la película La última Sillares, vol. 1, núm. 2, 2022 
diarias sobre la relevancia del filme, la canción que la musicalizaba, Sonny Boy, y el fenómeno que estaba causando en la capital del país. Estas notas entusiasmaron más al público, incitando a que asistieran a la función: "Cuando usted oiga 'La última canción' habrá sabido lo que vale la cinematografía parlante. Al Jolson le cautivará cantando 'Sonny Boy". ${ }^{19}$

La película se presentó en el Teatro Independencia, suponemos que por la capacidad de la sala. La empresa se expresó respecto a esta función:

Como siempre, EL GRAN CIRCUITO RODRÍGUEZ escribe una nueva página de arte en la historia de Monterrey. La empresa se siente como nunca una íntima satisfacción al presentar en esta progresista ciudad la primera película hablada, cantada y musicada. Después de la ciudad de México, toca a Monterrey la honra de admirar este nuevo espectáculo que está asombrando al mundo. La mejor recomendación que podemos hacer de este film, es que lo oigan. Los comentarios los dejamos al inteligente público que nos ha alentado para continuar nuestra tarea en pro del arte cinematográfico y teatral. ${ }^{20}$

Después del grandioso éxito y de los primeros aparatos parlantes instalados en el Teatro Independencia, el 5 de agosto, se presentó la segunda película Submarino (1929), de Columbia Pictures:

canción”, Monterrey, N.L., 26 de junio de 1929, 2

19 Periódico El Porvenir. "Cuando Ud. Oiga La última canción habrá sabido lo que vale la cinematografía parlante. Al Jolson le cautivará cantando", Monterrey, N.L., 6 de julio de 1929, 2.

20 Periódico El Porvenir. "La última canción", Monterrey, N.L. 25 de julio de 1929, 4.

Sillares, vol. 1, núm. 2, 2022

DOI: https://doi.org/10.29105/sillares1.2-4 
Todavía no nos pasa la impresión que nos produjera "La última canción", cuando los Hermanos Rodríguez, nos ofrecen otra tragedia tremenda de la cinematografía parlante... tenga usted la seguridad de que esta nueva película parlante habrá de gustarle por su novedoso argumento y sobre todo por la adaptación de los ruidos adaptados a la acción y la sincronización musical. ${ }^{21}$

La empresa abogó con distintas casas productoras para la exhibición de películas parlantes en la ciudad, como el filme Llegó la escuadra, pero debido a compromisos que tenía Warner Bros con otras empresas de la república, ésta no pudo acceder a los deseos de la Empresa A. Rodríguez. ${ }^{22}$ Sin embargo, se presentaron más películas parlantes, como Chantaje (s.f), de Paramount Pictures, el 11 de septiembre; Bohemios (1929), de Universal Pictures, el 5 de octubre; Alías Jimmy Valentine (1929), el 19 de septiembre, entre otras. Pero no solo se exhibían películas extranjeras. La empresa exhibió El Coloso de Mármol (1929), el 19 de septiembre, en el Gran Teatro Rodríguez, obra dirigida por Manuel R. Ojeda, con las actuaciones de Anita Ruíz y Carlos Villatoro. ${ }^{23}$

A la par de sus triunfos fílmicos, la empresa comenzó a expandir la reestructuración de los teatros y salas de cine

21 Periódico El Porvenir. "Mañana se estrena Submarino en el Gran Teatro Independencia", Monterrey, N.L., 4 de agosto de 1929, 2.

22 Periódico El Porvenir. "Clara Bow hace una película para jóvenes y viejos: Llegó la escuadra, se estrenará mañana en el Teatro Rodríguez”, Monterrey, N.L., 9 de agosto de 1929, 6.

23 Periódico El Porvenir. "Hoy se estrenará El Colosio de Mármol y Alías Jimmy Valentine en el Gran Teatro Rodríguez", Monterrey, N.L., 19 de septiembre de 1929, 6 .

Sillares, vol. 1, núm. 2, 2022

DOI: https://doi.org/10.29105/sillares1.2-4 
de la ciudad para instalar aparatos de sonido y exhibir filmes parlantes. El mismo año, durante el mes de diciembre, el Circuito Rodríguez contrató un equipo de RCA Photophone para equipar con toda la tecnología del momento el Gran Teatro Rodríguez. El ingeniero Clark, representante de la empresa RCA, junto a los regiomontanos el Ing. Fernando Balden y el electricista R. Buentello, fueron los encargados de montar el equipo de sonido. El presidente de la compañía mencionó que la instalación fue un éxito, comparando incluso el Teatro Rodríguez con los recintos de Broadway. ${ }^{24}$

La reinauguración del teatro se llevó a cabo el viernes 20 de diciembre de 1929, a las 8:15 de la noche, y se presentó un repertorio de diversas cintas sonoras para finalmente exhibir la esperada película de Alan Crosland, The Jazz Singer (1927). También, se presentó una filmación de Lupe Vélez, cantando canciones españolas como La canción del Lobo y la producción de Warner Bros, El Arca de Noé (1929). Ambos hermanos argumentaron:

Desde que iniciamos nuestra labor en el negocio cinematográfico-teatral, nuestros pasos se han encaminado hacia el firme propósito de mejorar en todo nuestro servicio. Somos servidores del público, justo es ir, a medida que el medio nos lo permite, presentando lo que anhela. De esta manera, ahora que el cine ha evolucionado enormemente en las grandes metrópolis estadunidenses, hemos querido ser de los primeros en introducir en

24 Periódico El Porvenir. "Gran Teatro Rodríguez", Monterrey, N.L., 20 de diciembre de 1929, 2.

Sillares, vol. 1, núm. 2, 2022

180

DOI: https://doi.org/10.29105/sillares1.2-4 
México esta nueva fase del cinema. Hoy vemos realizado uno de nuestros ideales, pues el RCA PHOTOPHONE que inauguramos en el Teatro Rodríguez, llena una precisa necesidad en el ambiente evolutivo del arte fílmico en Monterrey, ya que en una ciudad de la importancia de la nuestra es merecedora a tener más altos factores representativos de ese mismo arte. No obstante el elevado costo de estos aparatos no hemos vacilado un momento en adquirirlo con el deseo siempre vivo de complacer a nuestros favorecientes. Tenemos fe, tenemos confianza plena en que saldremos triunfantes de este nuevo ramo de la cinematografía que tiende a conquistar al mundo, porque conocemos bien el entusiasmo y cultura de que es poseedor el público regiomontano y por tal motivo continuaremos bregando necesariamente en nuestro medio para corresponder a los favoritos de ese mismo público. ${ }^{25}$

Durante la década de 1930, el Circuito Rodríguez se encargó de firmar contratos con empresas productoras estadounidenses, como Paramount Picture y algunas europeas para exhibir las mejores producciones en la ciudad. Incluso, comenzaron a equipar sus salas de cine foráneas para presentar películas sonoras gradualmente y mejorar sus proyectores con aparatos de fibra de oro de empresas nacionales como la R.C.A. Víctor Mexicana, S.A.

Un cambio significativo en las carteleras fue la propagación de películas nacionales, como el caso de Dios y Ley (1929) de Guillermo Calles, quien llegó a la ciudad para presentar su obra, el 23 de abril, en el Teatro Independencia. ${ }^{26}$

25 Periódico El Porvenir. "El Gran Teatro Rodríguez”, Monterrey, N.L., 20 de diciembre de 1929, 2.

26 Periódico El Porvenir. "Dios y Ley”, Monterrey, N.L., 23 de abril de 1930, 7. Sillares, vol. 1, núm. 2, 2022 
Dentro de los documentos del Archivo Histórico de Monterrey, se localizaron oficios por parte de otros estados de la república donde recomendaban a los empresarios no conceder licencia a aquellas películas vitafónicas habladas en inglés o cualquier otro idioma que no sea el español. ${ }^{27}$

Sin embargo, se puede apreciar en las carteleras que no se dejaron de presentar películas extranjeras, aunque la mayoría de ellas se presentaban de forma silente y otras solo musicalizadas sin diálogos. La empresa siempre abogó por la presentación de películas, aunque estas fueran censuradas a nivel nacional, pese a los constantes reclamos del gobierno municipal. Ese mismo año, se llevaron a cabo los trabajos para instalar equipos de sonido en el Teatro Lírico, Teatro Salón Variedades y Teatro Zaragoza, comenzando una expansión de las presentaciones de películas sonoras, a las cuales el público podía acceder sin problemas de falta de butacas o saturación del recinto. ${ }^{28}$

Se podría argumentar que, si en un inicio el cine compitió con el teatro, en esta nueva etapa su desarrollo no tuvo competencia. Incluso, la llegada del sonido aumentó la popularidad del espectáculo. Y aunque se seguían presentando obras teatrales, así como las famosas transmisiones de radio que ocuparon recintos para retransmitir programas y conciertos, el cine se convirtió en una de las actividades recreativas más

27 AHMM. Actas de Cabildo, Fondo Monterrey Contemporáneo, vol. 999, exp. 1930/002, 29 de julio de 1930, f.4.

28 Periódico El Porvenir. Monterrey, N.L., 19 de octubre de 1930, 4.

Sillares, vol. 1, núm. 2, 2022

DOI: https://doi.org/10.29105/sillares1.2-4 
importantes en la ciudad, o por lo menos así se refleja en la prensa local.

Las salas de cine se abarrotaban, llegando a presentarse quejas de personas que quedaban paradas en la función. Ante esto, el municipio llamó la atención a los empresarios para regular el cupo de los recintos, pues muchas personas pagaban el valor correspondiente por un lugar y al final terminaban de pie. ${ }^{29}$ Solo el Teatro Salón Variedades y el Teatro Independencia cumplían con el objetivo de contar con lugares separados. Por otro lado, la salida de los eventos también presentaba dificultades, pues el público de las salas ocasionaba aglomeraciones obstruyendo el tránsito. ${ }^{30}$

Se puede apreciar, también, que dentro de los recintos no se respetaban las normas "moralizadoras" o de "etiqueta". La emoción que despertaba la película en el público lograba silbidos, comentarios, gritos, entre otros, que para muchos sectores de la sociedad eran meras vulgaridades. Se propuso a los Rodríguez que hicieran de conocimiento al público algunas reglas o sugerencias, como: "La gente culta aplaude, no manifieste usted su ignorancia silbando en vez de aplaudir", entre otras semejantes, con el objetivo de que el público tuviera una "adecuada actitud" en los recintos. Por otro lado, al exterior de los mismos también comenzaron algunas complicaciones. La popularidad había aumentado aún

29 AHMM. Actas de Cabildo, Fondo Monterrey Contemporáneo, vol. 999, exp.1928/005, 17 de enero de 1928, f.6.

30 AHMM. Actas de Cabildo, Fondo Monterrey Contemporáneo, vol. 999, exp. 1930/023, 23 de septiembre de 1930, f. 12.

Sillares, vol. 1, núm. 2, 2022

DOI: https://doi.org/10.29105/sillares1.2-4 
más con la divulgación de los carteles que se instalaban fuera de los cines o en los espacios públicos, lo que a veces llegó a ser de gran molestia para otros sectores sociales, como los comerciantes, que donde quiera visualizaban los grandes y pequeños postes que daban mal aspecto. ${ }^{31}$

Las reacciones de la sociedad, sin embargo, parecieron favorables. El público se identificaba con las estrellas de cine hasta imitar los aspectos físicos de los actores. Algunos productos de higiene personal, como las pastas dentales, aprovechaban el fenómeno del cine para vender sus productos, argumentando que "todas las estrellas de la pantalla tienen dientes hermosos". ${ }^{32}$ Inclusive, se presentaban secciones de peinados, con el fin de que las damas de la época imitaran la moda de las actrices como Estelle Taylor o Raquel Torres:

Se hace con un simple forzal de la muñeca y una sencilla banda de cualquier tela. Pone cuidadosamente a través de la cabeza sostenido en ese lugar dos horquillas, una detrás de cada oreja. Esto hace que el pelo de atrás se mantenga unido y listo. Entonces, el cabello largo que es tan desagradable en la nuca se ensortija teniendo cuidado de que la banda quede oculta. ${ }^{33}$

Por otro lado, ante la crisis de 1929, cada estado del país había intentado aplicar sus propias soluciones. En Nuevo León, después

\footnotetext{
31 AHMM. Actas de Cabildo, Fondo Monterrey Contemporáneo, vol. 999, exp. 1928/005, 24 de enero de 1928, f.5.

32 Periódico El Porvenir. Monterrey, N.L., 12 de enero de 1928, 4.

33 Periódico El Porvenir. "Cómo debe usted peinarse Señorita, si quiere seguir la moda de las famosas estrellas de cine", Monterrey, N.L., 7 de julio de 1929, 8. Sillares, vol. 1, núm. 2, 2022

184

DOI: https://doi.org/10.29105/sillares1.2-4
} 
del intento de Sáenz por ocupar la presidencia y de designar a tres gobernadores interinos, Benítez, Calles y Chapa, diversos grupos locales apoyaron la candidatura del ingeniero en minas Francisco A. Cárdenas, viendo en él un intento de recuperar la economía local ante la crisis mundial.

Según Morado Macías, Cárdenas, que gobernó el estado entre 1931 y 1933, reconoció el impacto negativo de la crisis señalando que había afectado seriamente las finanzas públicas, por lo que su administración había diseñado una estrategia para generar ahorros significativos. También señaló que la crisis generó un aumento importante en las tasas de desempleo, tomando como medida la creación de un Comité pro-Desocupados con sede en Monterrey, que gestionaba empleos dentro de obras públicas. ${ }^{34}$

A este problema se añadía la situación de los repatriados. Debido a la crisis, miles de personas, quienes prestaban sus servicios como mano de obra, fueron expulsados de Estados Unidos. Monterrey era una de las primeras rutas tomadas por los migrantes, quienes llegaban sin alimentos, sin dinero, ni hogar. Para ello, Cárdenas solicitó ayuda del gobierno federal para erradicar este problema, solventando su pasaje para que los migrantes regresaran a su lugar de residencia. En diciembre de

34 César Morado Macías, "Los gobiernos de los generales de la Revolución mexicana", en Del Reino al Estado. Los gobernadores de Nuevo León: 15792017, ed. Romeo Ricardo Flores Caballero (Monterrey: Serna Impresos, 2019), 178.

Sillares, vol. 1, núm. 2, 2022

185

DOI: https://doi.org/10.29105/sillares1.2-4 
1933, Cárdenas renunció a la gubernatura, dejando el puesto a otro gobernador interino o sustituto, Pablo Quiroga.

Pero para el ramo de los espectáculos, la crisis fluyó sin mayores daños, sobre todo el cine. La prensa argumentaba que los cambios que presentó el cinematógrafo no sólo trajeron modificaciones en la forma de hacer y ver cine, sino, en la generación de nuevos empleos. ${ }^{35} \mathrm{Y}$ es que la expansión de las salas de cine, junto a las cantidades de películas que se estaban produciendo y que eran posteriormente exhibidas en los recintos de la ciudad, aumentaron el personal empleado por las empresas.

Era lógico que los hermanos no pudieran encargarse totalmente de todos los recintos a su cargo. Por ello, era importante contar con trabajadores que colaboraran en diversas áreas: algunos cumplían con la misión de exhibir los filmes, otros eran encargados de su distribución en los salones foráneos, otros del aseo, de las amenidades con que contaba el cine (dulcería, bar, entre otras), y otros de administrar el lugar.

Continuando con las exhibiciones, los hermanos siguieron programando y adquiriendo películas nacionales y habladas en español. Es de suponerse que esto se deriva de la gran producción nacional y que fue durante el periodo del "Maximato" en que el cine sonoro se consolidó en una etapa que ha sido llamada época preindustrial. El cine mexicano estaba intentando consolidarse

35 Periódico El Porvenir. "Notas cinematográficas: una ciudad dentro de otraAumentó el personal para las películas parlantes", Monterrey, N.L., 25 de diciembre de 1930, 4.

Sillares, vol. 1, núm. 2, 2022 
entre las grandes industrias cinematográficas. Solo durante 1932 a 1936 se realizaron aproximadamente 92 filmes, según datos de García Riera, de ficción, horror, arte, históricos, comedia e incluso se incursionó hacía nuevos géneros como el ranchero. ${ }^{36}$

Dentro de esta consolidación de la industria en México, se produjo la primera película sonora: Santa. La sociedad regiomontana estaba entusiasmada, e incluso los periodistas argumentaban que la industria cinematográfica seria y fuerte había quedado definitivamente establecida en el país y solo necesitaba un franco apoyo a fin de consolidarse bajo bases sólidas, fuertes. ${ }^{37}$

El 15 de abril de 1932, Santa se presentó en el teatro Rodríguez. La obra fue dirigida por Antonio Moreno y estuvo a cargo de la firma Compañía Nacional Productora de Películas. Para darle sincronía al sonido y la imagen, Santa contó con la colaboración de los hermanos Roberto y Joselito Rodríguez, hermanos del reconocido Ismael Rodríguez, empleando su más reciente invento, el Rodríguez Sound Recording System.

Se llevaron a cabo dos funciones, una a las 8:15 p.m. y otra a medianoche. Se invitó a la soprano Minerva Rodríguez para amenizar la velada, e incluso, gracias a las transmisiones de radio, se detallaron los momentos del evento para quienes no

36 Emilio García Riera, Breve historia del cine mexicano. Primer siglo, $1897-$ 1997 (México, DF: MAPA, 1998), 80.

37 Periódico El Porvenir. "La industria cinematográfica seria y fuerte ha quedado definitivamente establecida: Santa, la primera producción de la Compañía Nacional Productora de Películas se estrenará en el Gran Teatro Rodríguez el viernes 15 del actual", Monterrey, N.L., 13 de abril de 1932, 4.

Sillares, vol. 1, núm. 2, 2022 
pudieron asistir, consiguiendo que Lupita Tovar se comunicara por dicha vía para saludar a los concurrentes afortunados. Santa sólo se programó en el Teatro Rodríguez por razones de contrato y el equipo de sonido con que éste contaba. ${ }^{38}$

\section{Conclusiones}

Aunque al inicio se creyó que su éxito sería momentáneo, durante la década de 1920 se comprobó que el cine fue tomando impulso: se crearon salas en diversas zonas de la ciudad, se creó un público específico para el espectáculo, comenzó una fuerte distribución fílmica, entre otros aspectos que dejaron clara su importancia.

Durante dicha época, comenzaron a destacar cambios en el espectáculo -la adaptación del sonido a la imagen-, mismos que provocaron una coyuntura entre la relación de éste con la sociedad. El cine sonoro se convirtió en un fenómeno internacional y en diversas partes del mundo fue recibido con entusiasmo. Esta revolución sonora comenzó su expansión en el país, incluyendo a Monterrey. Sin embargo, no todos los círculos sociales aceptaron los cambios.

La recopilación de información indica que la sociedad regiomontana se rehusaba a los cambios que presentó el espectáculo. Esto se puede observar desde su arribo, donde algunos círculos sociales consideraban al cine como una atracción vulgar; posteriormente, también hubo resistencia hacia el cambio de vistas móviles a películas con argumentos, pues estaban

38 Periódico El Porvenir. "Santa", Monterrey, N.L., 15 de abril de 1932, 4. Sillares, vol. 1, núm. 2, 2022 
acostumbrados a películas más simples como paseos cotidianos, salidas de fábricas, paisajes, entre otros.

Dichos cambios causaron disgusto social en su momento, sin embargo, la sociedad llegó a adaptarse. Con el arribo del sonido hubo reacciones similares. Algunos círculos sociales, principalmente los intelectuales, estaban en contra, cuestionando los constantes cambios del espectáculo. Por otro lado, la sociedad en general estaba entusiasmada por las nuevas tecnologías, abarrotando los recintos para escuchar hablar o cantar a sus artistas favoritos.

El arribo del sonido implicó cambios en la forma de ir al cine. Su aparición en la ciudad orilló a los empresarios a modificar la estructura de distribución, exhibición, e incluso a modificar las antiguas salas de cine para instalar los aparatos sonoros. Esto provocó que para la sociedad de clase media o baja fuera más complicado acceder a esta atracción, ya que los gastos realizados para hacer estos cambios se vieron reflejados en las costosas entradas.

\section{Referencias}

\section{Archivo}

Archivo Histórico Municipal de Monterrey

\section{Bibliográficas}

García Riera, Emilio. Breve historia del cine mexicano. Primer siglo, 1897-1997. México, DF: MAPA, 1998.

Sillares, vol. 1, núm. 2, 2022 
Maltby, Richard. Explorations in New Cinema History: Approaches and Case Studies. Londres: Blackwell Publishing Ltd, 2011.

Meers, Philippe. "Metodologías de investigación para la 'nueva Historia del cine"”. En Miscelánea sobre el entorno audiovisual, editado por Francisco Ubierna Gómez y Javier Sierra Sánchez. España: Fragua, 2014.

Morado Macías, César. "Los gobiernos de los generales de la Revolución mexicana". En Del Reino al Estado. Los gobernadores de Nuevo León: 1579- 2017, editado por Romeo Ricardo Flores Caballero. Monterrey: Serna Impresos, 2019.

Segovia, Rafael, y Alejandra Lajous. Historia de la Revolución mexicana, período 1928-1934: Los inicios de la Institucionalización, la política del Maximato. México, DF: El Colegio de México, 1978.

Sifuentes Zúñiga, Kassandra. "Implicaciones sociales y culturales relativas al desarrollo del cine en Monterrey, 18981927”. Universidad Autónoma de Nuevo León, 2017.

Vidal Bonifaz, Rosario. "Los inicios del cine sonoro y la creación de nuevas empresas fílmicas en México (1928-1931)". Revista del Centro de Investigaciones, núm. 29 (2008): 17-28. 